\title{
Modeling of Immunosenescence and Risk of Death from Respiratory Infections: Evaluation of the Role of Antigenic Load and Population Heterogeneity
}

\author{
T. E. Sannikova ${ }^{1 *}$, A. A. Romanyukha ${ }^{1}$, E. Barbi $^{2}$, G. Caselli $^{2}$, C. Franceschi ${ }^{3}$, A. I. \\ Yashin $^{4}$ \\ ${ }^{1}$ Institute of Numerical Mathematics, Gubkina 8, 119991, Moscow, Russia \\ ${ }^{2}$ Department of Statistical Sciences, Sapienza University of Rome, Italy \\ ${ }^{3}$ Dipartimento di Medicina Specialistica Diagnostica e Sperimentale (DIMES) \\ University of Bologna, Italy \\ ${ }^{4}$ Biodemography of Aging Research Unit, Duke University, Durham, USA
}

\begin{abstract}
It is well known that efficacy of immune functions declines with age. It results in an increase of severity and duration of respiratory infections and also in dramatic growth of risk of death due to these diseases after age 65 . The goal of this work is to describe and investigate the mechanism underlying the age pattern of the mortality rate caused by infectious diseases and to determine the cause-specific hazard rate as a function of immune system characteristics. For these purposes we develop a three-compartment model explaining observed risk-of-death. The model incorporates up-to-date knowledge about cellular mechanisms of aging, disease dynamics, population heterogeneity in resistance to infections, and intrinsic aging rate. The results of modeling show that the age-trajectory of mortality caused by respiratory infections may be explained by the value of antigenic load, frequency of infections and the rate of aging of the stem cell population (i.e. the population of T-lymphocyte progenitor cells). The deceleration of infection-induced mortality at advanced age can be explained by selection of individuals with a slower rate of stem cell aging. Parameter estimates derived from fitting mortality data indicate that infection burden was monotonically decreasing during the twentieth century, and changes in total antigenic load were gender-specific: it experienced periodic fluctuations in males and increased approximately two-fold in females.
\end{abstract}

Keywords and phrases: immunosenescence, respiratory infections, individual hazard

Mathematics Subject Classification: 91D20, 92C42, 93A30

\section{Introduction}

Aging of the immune system, as well as aging of the entire organism, occurs at all levels of its hierarchy. It occurs at the level of cells - the telomeres of $\mathrm{T}$ cells shorten with age, which results in a decrease in immune reaction rates. At the level of cell population - a decrease in the number of naïve $\mathrm{T}$ cells and an increase in the number of $\mathrm{T}$ memory cells take place. At the level of the whole immune system the volume of the peripheral lymphoid tissue shrinks with increasing age. All these processes lead to a decrease in the immune function efficacy and an increase in the frequency and the severity of infections, and an increase in the chances of death from infectious diseases with age.

${ }^{*}$ Corresponding author. E-mail: te_san@yahoo.com 
Immunosenescence may contribute to development of many other health disorders. Zhu et al revealed that viral and bacterial infections could have an influence on development of cardiovascular diseases $[43,44]$. The authors found that increasing pathogen burden is significantly associated with increasing risk of coronary artery disease and myocardial infarction. Cawthon et al found that deaths from heart disease were significantly more frequent in patients with short telomere lengths of blood cells' DNA than in those with long telomere lengths [8]. These indicate that aging of immune system may be associated not only with increased proportion of deaths from infectious diseases among the elderly, but also with other major causes of death in developed countries $[6,17]$.

A study of the immune system of healthy centenarians [13,34] revealed that several immune parameters of these individuals were well conserved and close to normal values for age 50 . There exists a great deal of evidence that the rate of immunosenescence can vary widely [35]. The sources of such variation have genetic and environmental origin, however neither factors nor mechanisms of regulating immunosenescence are exactly known. One can suspect an important role of antigenic load, first in development, and then in deterioration of the immune system during the life course [14,25,28,29]. The evidence is accumulating that chronic and acute diseases could accelerate immunosenescence [30,35, 41]. Modification of the immune state using vaccination, antiviral and hormonal therapies, stem cell transplantation - and possibly by regulation of telomerase activity $[2,11]$ - could slow down processes associated with immunosenescence. Results of such interventions are determined by several processes, among which the processes maintaining long-term homeostasis of lymphocyte concentration play a crucial role. During immune response to infection, the population of specific lymphocytes rapidly grows and increases by 5-6 orders of magnitude. After pathogen elimination, $95-99 \%$ of the specific lymphocytes die within 10-20 days; the rest survive and replenish the pool of memory cells. The capacity of the immune system to support the population of T lymphocytes is suggested to be limited by the volume of peripheral lymphoid tissue; therefore newly produced memory cells accelerate death of existing memory cells. Vaccinations and application of immunostimulators contribute to this effect. High activity of the thymus also reduces the duration of protective immunity since new naïve cells crowd out memory cells [15].

Process of pathogen transmission within a population is traditionally considered by epidemiologists [16, $19,26,42]$. Host's resistance is an important epidemiological factors and its changes are determined by efficacy of host immune system. Therefore, it is important to examine the impact of immunosenescence on epidemiology of respiratory infections and related mortality.

In this paper we assume that an increase in the mortality from infectious diseases in human cohorts with age is associated with the aging related decline in immune system functioning, and investigate the mechanisms underlying the relation between the risk of death from respiratory infections and immunosenescence. For this purpose we developed a model of immunosenescence and its connection to the risk of death from respiratory infections (MIRD). We use this model to fit data on deaths caused by bronchitis, pneumonia and influenza for Italian cohorts born between 1873 and 1895 [5]. As a result we obtain and interpret the time trajectories of indices describing changes in antigenic load, frequency of infections, rate of telomere erosion in T-lymphocyte progenitor cells in different ages.

\section{Model of immunosenescence and risk of death from respiratory infections (MIRD)}

The MIRD consists of three compartments (Fig. 1). The first compartment is a model of immunosenescence. It describes age related changes in immune system characteristics. The second model makes it possible to estimate the severity of infectious disease for different states of the immune system. The state of the immune system is determined by the concentration and replicative capacity of naive and memory $\mathrm{T}$ cells. These properties determine the rate of immune response to infection. The third compartment of MIRD is a function describing population heterogeneity in resistance to infections. Multiplied by the frequency of respiratory infections, it yields the estimated risk of death from the diseases in the population.

\subsection{Model of immunosenescence}

The mathematical model of immunosenescence [31,32] describes age-related changes in a population of peripheral $\mathrm{T}$ cells. These changes could be subdivided into two groups - determined by genetic and 


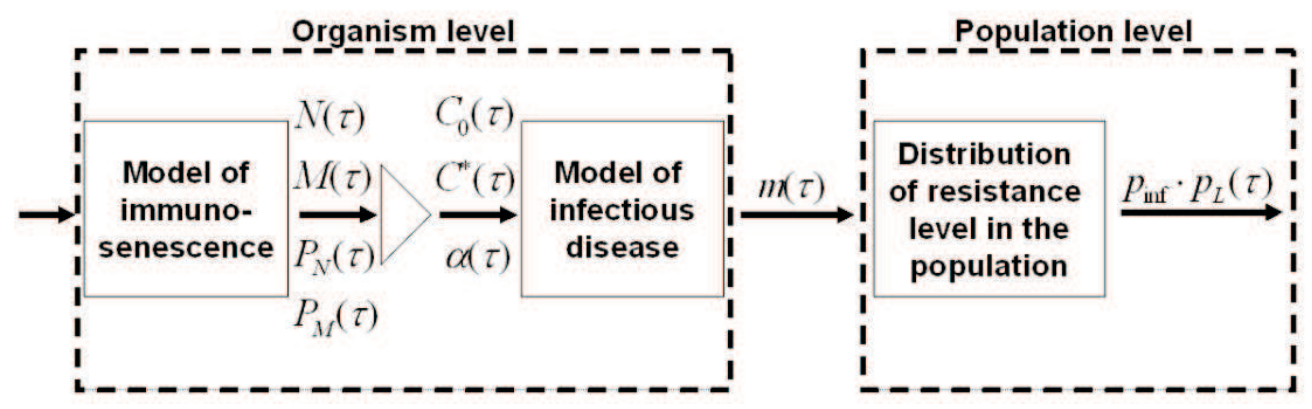

FigURE 1. Flow chart of the mathematical model of immunosenescence and risk of death from respiratory infections (MIRD).

environmental factors. The former group contains shortening of telomere length in stem cells, thymus involution and diminishing of the peripheral lymphatic tissue. The rates of the processes are presumed to be intrinsic (heritable) properties of the individuals.

The following processes form the latter group

- a decrease in the concentration of naïve T cells in the intact peripheral lymphoid tissue $(N(\tau))$;

- an increase in the concentration of memory $\mathrm{T}$ cells $(M(\tau))$; and

- a shortening of the length of DNA telomeres in naive and memory T cells $\left(P_{N}(\tau)\right.$ and $P_{M}(\tau)$, respectively).

Their rates depend on antigenic load, which includes both infectious and noninfectious antigens. The model of immunosenescence is a system of nonlinear differential equations:

$$
\begin{aligned}
\frac{d N}{d \tau} & =\frac{N^{*}}{V}-\alpha_{1} \frac{L}{V} N-\mu_{N} N-\frac{d V}{d \tau} \frac{N}{V}, \\
\frac{d M}{d \tau} & =\rho_{1} \alpha_{1} \frac{L}{V} N+\rho_{2} \alpha_{2} \frac{L}{V} M+\mu_{M}\left(C^{*}-N-M\right)-\frac{d V}{d \tau} \frac{M}{V} \\
\frac{d P_{N}}{d \tau} & =\left(P^{*}-P_{N}\right) \frac{N^{*}}{N V}, \\
\frac{d P_{M}}{d \tau} & =\rho_{1} \alpha_{1}\left(P_{N}-P_{M}-\lambda_{N}\right) \frac{L}{V} \frac{N}{M}-\left(\rho_{2}+1\right) \alpha_{2} \lambda_{M} \frac{L}{V} .
\end{aligned}
$$

The first equation describes changes in the concentration of the naïve T cells with age $(\tau)$. The first term characterizes the influx of naïve T cells from the thymus. Since the volume of functioning tissue in thymus, which determining level of $N^{*}$ might decrease to the undetectable level without serious consequences for the health [37] we can describe the age dynamics of the rate of naïve $T$ cells influx as

$$
N^{*}(t)=N_{0}^{*} e^{-k_{T} t}
$$

Under the action of antigenic load, the number of naïve $\mathrm{T}$ cells decreases (the second term), and the number of memory $\mathrm{T}$ cells increases (the first term of the second equation). The antigenic stimulation also causes proliferation of memory $\mathrm{T}$ cells, which is described by the second term of the second equation. Here $L$ characterizes antigenic load. It is supposed to be constant during the lifetime. In the context of the model, the value of antigenic load determines rates of consumption of naïve $\mathrm{T}$ cells and expansion of memory cells.

The third equation describes the dynamics of average telomere length $P_{N}$ in naïve T cells. Here $P^{*}$ is the telomere length in cells that have recently left the thymus. The length of telomere in new naïve $\mathrm{T}$ cells cannot be less then Heyflick limit, $P_{m i n}$, so it decreases with age as

$$
P^{*}(t)=\left(P_{0}^{*}-P_{\text {min }}\right) e^{-k_{P} t}+P_{\text {min }} .
$$

The last equation describes the changes in average telomere length in memory $\mathrm{T}$ cells. The rate of this process is determined primarily by the difference between average length of telomere in naïve and 


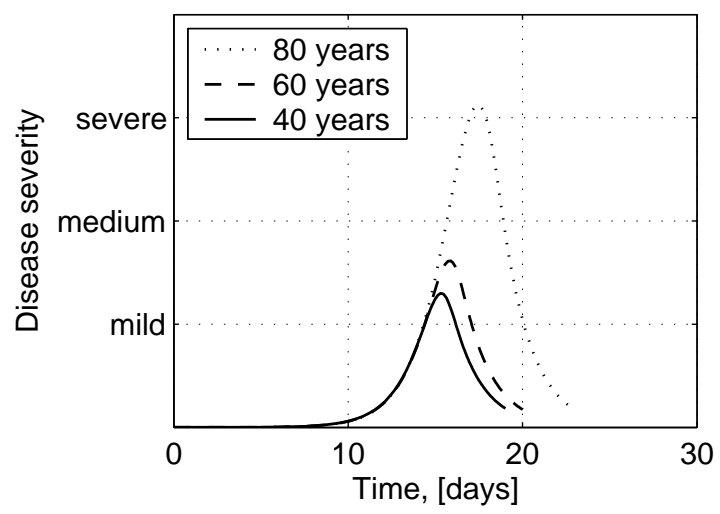

FiguRE 2. Infection-induced damage of tissues in the course of disease for different ages. On the vertical axis - the fraction of destroyed cells in target organ. Disease severity is defined as a maximum of tissue damages [24].

memory cells minus the average telomere loss during immune response. It is also proportional to the antigenic load.

The volume of the intact peripheral lymphoid tissue decreases with age as

$$
V(t)=\left(V_{0}-V_{\min }\right) e^{-k_{V} t}+V_{\min }
$$

and compensates for the decline of the total number of $\mathrm{T}$ cells.

The parameters values and initial conditions for the system (0.1-0.7) are given in Appendix 1.

\subsection{Model of infectious disease}

This mathematical model describes the processes that determine beginning, course and outcome of any infection: invasion and propagation of the pathogen, infection-induced damage of tissues, immune response, elimination of the pathogen and tissue regeneration [22]. Model parameters are the rates of these processes. The values of the parameters determine severity and outcome of the modeled disease. Hence, age-related changes in disease severity (and therefore the risk of lethal outcome) can be evaluated as a result of the varying rate of immune response according to the age trend.

In Fig. 2 the extent of tissue damage in the course of disease is presented for different ages. Disease severity is defined by a maximum of tissue damage. The higher the disease severity is, the higher the risk of lethal outcome. Detailed description of the model's equations, variables and parameters are given in Appendix 2.

\subsection{Relationship between disease severity and the risk of death}

The conjunction of the model of immunosenescence (0.1-0.7) and the model of infectious disease (0.90.12 ) allows us to model the age related increase in the disease severity for an average individual. In order to execute the transition to the population level we should take into consideration the fact that the individual probability of death from respiratory infection at age $\tau$ depends on probability of getting infected $\left(p_{\text {inf }}\right)$ and probability of death from certain tissue damage $\left(p_{L}\right) \cdot p_{\text {inf }}$ and $p_{L}$ are supposed to be i.i.d. and therefore, the required probability of death from respiratory infections can be defined as a product of $p_{\text {inf }}$ and $p_{L}$. The probability of getting infected is supposed to be a characteristic of the environment and might vary with time and location [33]. So, this parameter is calculated while the data on the cohort mortality are fitted. The probability of death having certain volume of damaged tissues was determined on the base of the clinical study [23]. Having analyzed 600 cases of acute pneumonia the authors concluded that $4 \%$ of the patient died when only $10 \%$ of the lung tissues were destroyed and a half of the patients survives if the pneumonia attacked $35 \%$ of lung tissue. Hence, the probability of death at the volume of damaged tissues $m$ can be defined as a cumulative distribution function with mean value 


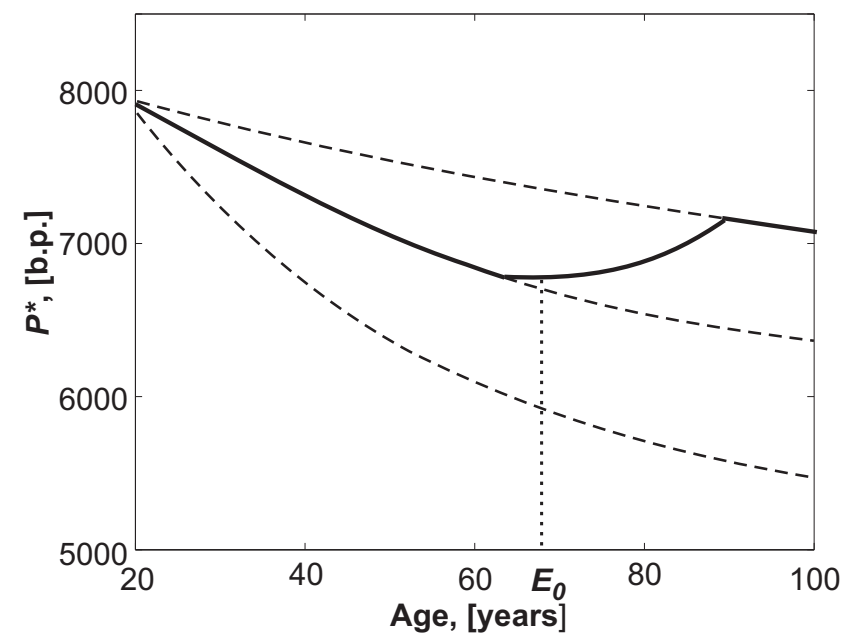

FiguRE 3. The average length of the telomeres calculated among alive individuals can increase since the selection favored those with slow rate of aging (hypothetical plot). $P^{*}$ is the telomere length in the progenitor of naïve cells. The upper dotted line shows the telomere length in the subpopulation with slow immune system aging. The lowest dotted line corresponds to the fast aging subpopulation. The solid line describes the changes of average telomere length in the progenitor of naïve cells of the whole population. It is supposed that after age equal to the life expectancy at birth $\left(E_{0}\right)$ the cohort consists of the individuals with low rate of telomere shortening.

$m=0,35$.

$$
p_{L}(m)=\Phi(m)=\int_{0}^{m} \frac{1}{\sigma \sqrt{2 \pi}} e^{(t-a)^{2}} d t
$$

The values of the parameter were estimated based on clinical observations [23].

Thus, by means of the model of infectious disease and expression (0.8), the relationship between immunosenescence and risk of death could be established.

\subsection{Mortality from respiratory infections in the heterogeneous population}

It is a well-known fact that it is quite difficult to get a numerical estimate of immune defense efficiency but indirect evidence such as data on mortality from respiratory infections might be received. The left panel of Fig. 4 represents shaded contour maps of age-specific probabilities of death from respiratory infections for 23 male and 23 female cohorts born between 1873 and 1895 in the range of age from 50 to 98 . The maps offer a panoramic view of the male and female evolution of mortality over cohorts. The shading goes from white to black as the surfaces rise from low to high mortality. These cohorts faced the most dramatic events in history: wars, introduction of antiseptics and antibiotics, industrialization and urbanization. The difference in life expectancy at birth between the former and the latter cohort is more than 10 years [7]. Therefore it can be concluded that the earlier cohorts experienced more selection pressure than the later ones. As it was shown in [38] the selection might influence the shape of curve of cohort characteristics in different way. For the sake of accuracy the selection in the heterogeneous cohort should be taken into account.

Analysis of the mathematical model of immunosenescence (0.1-0.7) revealed that the strength of immunity at advanced age strongly depends on the rate of telomere erosion in T-lymphocyte progenitor cells accumulated during the life course. The telomere length in the progenitor of naive cells was assumed to be prone to the pressuer of the selection. For example, it was shown that individuals with shorter telomere lengths had a higher all-cause mortality rate, as well as a higher mortality rate from infectious diseases [8]. Hence, it is reasonable to assume that deceleration of mortality rates at old ages is related (attributable) to the selection of individuals with slower rates of telomere shortening in stem cells. It is then that the average rate of telomere erosion in T-lymphocyte progenitor cells in the cohort is supposed 
to decelerate. This hypothesis was tested using data on mortality rates from respiratory infections in Italian cohorts born between 1873 and 1895 .

Simulation of MIRD is a sequence of numerical calculation of each part of the model. First of all, the set of equations (0.1)-(0.7) describing the process of immunosenescence is solved by using Matlab solver for non-linear differential equation. The parameters values and initial values of the variables can be found in the table 1 (Appendix 1).

Secondly, for ages $\tau=20,21 \ldots 98$ the course of respiratory disease is simulated by means of the model (0.9)-(0.12) (Appendix 2) with the parameter values given in Table 2.. The initial value of the variable describing the concentration of immune cells is defined by the numerical decision of the previous model as far as the rate of their proliferation (see formula (0.14), (0.15)). The greatest value of the variable $m_{C}$ representing the fraction of damaged tissues gives us the severity of the disease for age $\tau$. It should be noticed that the system of differential equations (0.9)-(0.12) is solved by Matlab procedure for stiff problems.

Thirdly, using the function of probability of death for each severity and multipling it by probability of getting infected $\left(p_{\text {inf }}\right)$ the probability of death from respiratory infection at age $\tau$ is calculated.

Let us assume that the population cohorts can be divided into two sub-cohorts: with slow and fast rates of immune system aging represented by the rate of decline of average telomere length in respective sub-cohort. Then the rate of immune system aging of the entire cohort before age of $E_{0}$ is equal to the population average. Because of mortality selection process those with shorter telomeres (sub-cohort with fast rate of aging of immune system) will die first. Therefore the average age trajectory of the telomere lengths in T-lymphocyte progenitor cells will show decelerated rate of decline in telomere length (Fig. 3).

In the model (0.1-0.7), the decrease in telomere length in the progenitors of naïve cells is described by expression (0.6), where $k_{p}$ is the average rate of aging in population. Then age-related changes in the rate of telomere shortening in the progenitors of naïve cells $k_{p}$ related to the population heterogeneity could be specified by the following formula

$$
k_{p}(\tau)=\frac{k_{p 1}+k_{p 2}}{2}-\frac{k_{p 1}-k_{p 2}}{2} \cdot \frac{e^{v\left(\tau-E_{0}\right)}-e^{-v\left(\tau-E_{0}\right)}}{e^{v\left(\tau-E_{0}\right)}+e^{-v\left(\tau-E_{0}\right)}},
$$

where $k_{p 1}$ is the mean rate of telomere shortening in the progenitors of naïve cells in the initial population and $k_{p 2}$ is the mean rate in the long-lived population.

\section{Results}

We fit the life-table data on probability of death caused by respiratory infectious for male and female Italian cohorts born between 1873 and 1895. There is good agreement between the model and the data sets for medium and large values of the death rate (Fig. 4). In the case of small values (less then 0.005), the estimated risk of death is higher then observed. To provide a good fit, four parameters of the model were estimated for every cohort: two characteristics related to the environment (antigenic load and the frequency of respiratory infections) and heritable characteristics (the rates of telomere shortening in stem cells).

It can be seen (Fig. 5a) that antigenic load for males did not change much during this period of time. In females the antigenic load in 1873 was lower than that in males and increased with the year of birth of the cohort. The frequency of respiratory infections declines with year of birth for both sexes (Fig. 5b). Note that the data show that progress in reduction of male mortality from infections is slower than that of females. This observation is in agreement with slower progress in reduction of total mortality in males compared to females [40]. Here faster progress in reducing female mortality is reflected by faster decline in the frequency of respiratory infections in females.

Estimated rates of telomere erosion in T-lymphocyte progenitor cells are represented in Fig. 6. Fig. 6a shows the average rate of aging at young and middle ages for male and female cohorts. The estimated rates of stem cell aging in old age for men and women are shown in Fig. 6b. One can see that the rate of aging in old age is approximately five times less than in young and middle ages. In our opinion, this can be a consequence of greater probability of death among individuals with higher rates of stem cell aging.

Analysis of the trends reveals that female cohorts experienced a constant decrease in the rate of stem cell aging at young and middle ages - and an increase of the rate of aging in old age. This rearrangement (alteration) is probably accounted for by the significant increase in life expectancy (life span): from 46 to 


\section{Females}
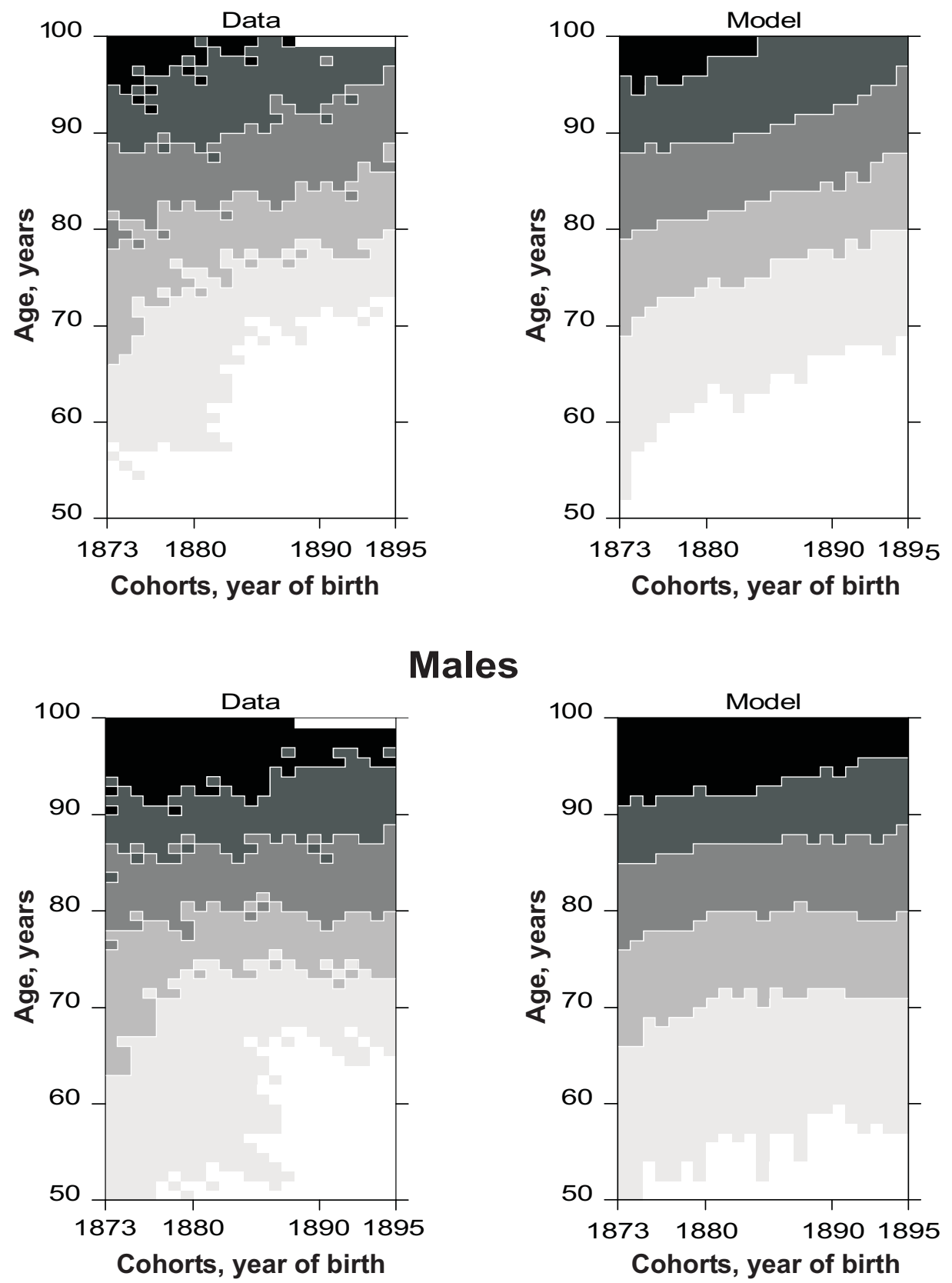

Probability of death caused by bronchitis, pneumonia and influenza:

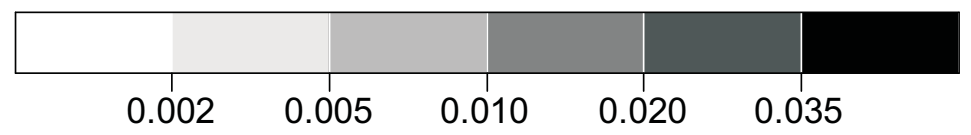

FIGURE 4. Shaded contour maps [39] of age-specific probabilities of death caused by bronchitis, pneumonia and influenza for Italian cohorts born between 1873 and 1895 . Comparison between model(right panel) and data(left panel). Calendar year is located on horizontal axis and the age - on the vertical axis. 
a)

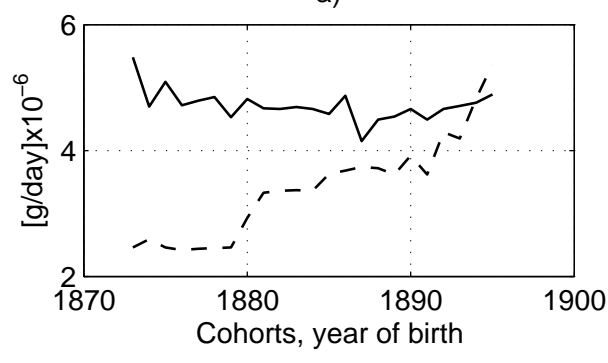

b)

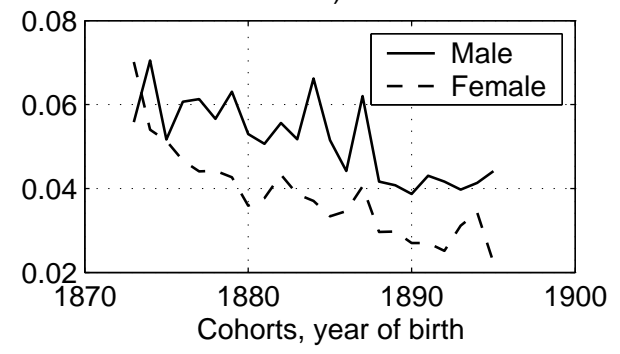

FIGURE 5. Estimated dynamics of environmental conditions which influenced immunosenescence in Italian cohorts born between 1873 and 1895. a) Antigenic load. b) Frequency of infections.

a)

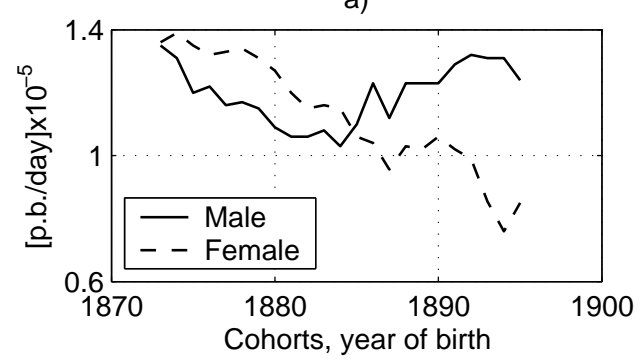

b)

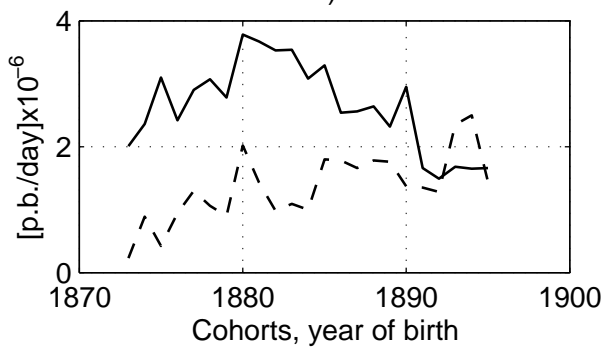

FiguRE 6. Estimated rates of telomere erosion in T-lymphocyte progenitor cells in Italian cohorts born between 1873 and 1895: a) in young and middle age, b) in old age.

55 years. An additional factor that decelerates aging of T-lymphocyte progenitor cells could be a decrease in frequency of infections (Fig. 5b). Then the increase in the rate of stem cell aging in the older group is caused by inflow of large numbers of individuals with relatively higher rates of aging.

It is interesting that we found no such pattern in male cohorts: the frequency of infections decreased to a lesser extent and experienced fluctuations; changes in the rates of stem cell aging showed no trend.

\section{Discussion}

The proposed model describes the relation between physiological and demographic aging. There is some evidence that replicative senescence of $\mathrm{T}$ cells results in growth of mortality caused by respiratory infections $[1,10,27]$. Clinical and experimental data show that exhaustion of $\mathrm{T}$ cell immunity is responsible for an age-related increase in severity and duration of viral infections. Severe viral infections at advanced age often induce severe and fatal bacterial infections, since replicative senescence of $\mathrm{T}$ cells is strongly associated with mortality from bacterial infections.

According to the proposed model, disease severity is determined by the concentrations of $\mathrm{T}$ cells and by the length of their DNA telomeres. After age 60, the course and outcome of infectious disease are highly influenced by the length of $\mathrm{T}$ cell telomeres. The existence of an association between higher risk of death from infectious disease and shorter telomere length was shown [8]. This association is masked by the genetically determined diversity of telomere length in the population. As shown in a twin study, [36] the heritability of telomere length accounts for $78 \%$; the other $22 \%$ is attributable to environmental factors. At the same time, there is some evidence that heritability of lifespan does not exceed 35\% [18,21]. The proposed model is an attempt to comprehend the roles of genetics, individual immune history and environment in longevity.

In our model environmental pressure on the immune system is characterized by the parameter of antigenic load which includes infectious load and intake of non-infectious antigens contained in food 
and air. The infectious load includes acute and chronic infections and can be defined as a mean rate of pathogen invasion in the organism. The value of this parameter characterizes the efficiency of immune defense in coping with infectious contamination.

As mentioned above, the antigenic load did not change much in males (Fig. 5a). In female cohorts born before 1879 the antigenic load was almost constant and 2.5 times less than that in male cohorts. For female cohorts born after 1879 the value of this parameter increased with year of birth, in other words the antigenic load is higher in those cohorts who were 19-35 years old in 1915. Italy, during the Great War (1915-1918), was still a very poor and culturally backward country. The industrial revolution did not take off until the beginning of the new century and was mainly limited to the major cities in the Northwest and the surrounding areas. Agriculture was still the main activity throughout the country and women, when the men left for the front, had to take on the extra burden of the hard labour in the fields and cover the industrial occupations abandoned by their men in order to support as well as care for their numerous offspring. Families at the time were numerous, social and health structures almost non-existent, public hygiene and in the home extremely lacking and nutrition poor. Women found it hard to reconcile their domestic chores with work outside the house, often paid at bare subsistence levels. The effects of poverty and malnutrition caused mortality levels that mirrored those of the ancient regime, both for women and for children. Indeed, the number of women at home who died more or less equalled that of the men who were at war and they were particularly struck by the Spanish flu epidemic, being more frail and malnourished that the men at the front $[3,4]$. Since this process was accompanied by a decrease in frequency of infections (Fig. 5b) it can be suggested that infectious load was increasing due to growth of incidences of chronic diseases.

The rate of telomere erosion in stem cells is an important biological characteristic determining life span of an individual. It is determined both by genetics and life history $[9,12,20,36]$. For females the rate of stem cell aging in young and middle ages was decreasing with year of birth (Fig. 6a), for males this characteristic was decreasing for cohorts born between 1873 and 1884 and increasing for almost all cohorts born between 1885 and 1895. A female organism is designed for child-bearing and it is natural to assume that this process is accompanied by excessive load on stem cells and accelerated shortening of their telomeres. Greater growth of the life expectancy in females compared to males could be caused by the decrease of the rate of stem cell aging which was due to significant changes in life style that affected child-bearing function and its realization (number of pregnancies and deliveries).

The proposed model establishes the relationship between physiological adaptation to antigenic load and age-specific mortality from infectious diseases. The approach developed allows for estimation of individual risk of death caused by infection and analysis of the impact of the environment on immunosenescence.

\subsection{Appendix 1.}

\subsection{Appendix 2. Model of infectious disease}

This mathematical model describes [22] the processes which determine beginning, course and outcome of all infections: invasion and propagation of the pathogen, infection-induced damage of tissues, immune response, elimination of the pathogen and tissue regeneration. Variables of the model are

$K(t)$, the concentration of the pathogen in target tissue;

$C(t)$, the concentration of specific lymphocytes in draining lymph nodes;

$F(t)$, the concentration of specific antibodies in blood;

$m_{c}(t)$, the fraction of target cells destroyed by pathogen.

The dynamics of immune response is described by the system of four differential equations

$$
\begin{aligned}
\frac{d K}{d t} & =\beta K-\gamma F K \\
\frac{d C}{d t} & =\alpha\left(1-m_{c}\right) F K-\mu_{c}\left(C-C^{*}\right), \\
\frac{d F}{d t} & =\rho C-\eta \gamma F K-\mu_{f} F \\
\frac{d m_{c}}{d t} & =\sigma\left(1-m_{c}\right) K-\mu_{m} m_{c} .
\end{aligned}
$$


TABLE 1. Initial conditions and model parameters for simulation of the normal aging of the immune system

\begin{tabular}{|c|c|c|}
\hline Parameter & Physical meaning and dimension & Estimate \\
\hline$\alpha_{1}$ & $\begin{array}{l}\text { Coefficient of sensitivity of naïve } \mathrm{T} \text { cells to antigen } \\
\text { load }\left(\mathrm{mlg}^{-1}\right)\end{array}$ & $1.5 \cdot 10^{4}$ \\
\hline$\alpha_{2}$ & $\begin{array}{l}\text { Coefficient of sensitivity of memory } \mathrm{T} \text { cells to antigen } \\
\text { load }\left(\mathrm{ml} \mathrm{g}^{-1}\right)\end{array}$ & $1.5 \cdot 10^{4}$ \\
\hline$\mu_{N}$ & $\begin{array}{l}\text { Death rate of naïve } \mathrm{T} \text { cells in the absence of antigen } \\
\text { load }\left(\text { day }^{-1}\right)\end{array}$ & $1.8 \cdot 10^{-4}$ \\
\hline$\mu_{M}$ & Death rate of memory $\mathrm{T}$ cells $\left(\mathrm{day}^{-1}\right)$ & 0.05 \\
\hline$\rho_{1}$ & Number of memory $\mathrm{T}$ cells produced by one naïve cell & 100 \\
\hline$\rho_{2}$ & $\begin{array}{l}\text { Number of memory } \mathrm{T} \text { cells produced by one memory } \\
\text { cell }\end{array}$ & 1.1 \\
\hline$\lambda_{N}$ & $\begin{array}{l}\text { Length of telomere repeats lost during transformation } \\
\text { of naïve cells to memory cell (bp cell }{ }^{-1} \text { ) }\end{array}$ & 1400 \\
\hline$\lambda_{M}$ & $\begin{array}{l}\text { Length of telomere repeats lost during self replication } \\
\text { of memory cells (bp cell }{ }^{-1} \text { ) }\end{array}$ & 500 \\
\hline$M^{*}$ & $\begin{array}{l}\text { Low limit for normal concentration of memory } \mathrm{T} \text { cells } \\
\text { in lymphoid tissue }\left(\text { cell } \mathrm{ml}^{-1}\right)\end{array}$ & $2.5 \cdot 10^{9}$ \\
\hline$k_{T}$ & $\begin{array}{l}\text { Rate of diminishing of naïve } \mathrm{T} \text { cells production with } \\
\text { age }\left(\text { day }^{-1}\right)\end{array}$ & $1.1 \cdot 10^{-4}$ \\
\hline$k_{V}$ & $\begin{array}{l}\text { Relative rate of reduction of lymphoid tissue volume } \\
\text { with age }\left(\text { day }^{-1}\right)\end{array}$ & $2.7 \cdot 10^{-5}$ \\
\hline$k_{P}$ & $\begin{array}{l}\text { Relative rate of the telomere repeats reduction in the } \\
\text { progenitor of naïve } \mathrm{T} \text { cells }\left(\mathrm{bp} \text { day }^{-1}\right)\end{array}$ & $1 \cdot 10^{-5}$ \\
\hline$L$ & Antigen load $\left(\mathrm{g} \mathrm{day}^{-1}\right)$ & $1 \cdot 10^{-6}$ \\
\hline$N_{0}^{*}$ & $\begin{array}{l}\text { The rate of } \mathrm{T} \text { cell production by the thymus at the } \\
\text { age of } 18 \text { (cell day }^{-1} \text { ) }\end{array}$ & $4 \cdot 10^{8}$ \\
\hline$V_{0}$ & Volume of lymphoid tissue at the age of $18(\mathrm{ml})$ & 1500 \\
\hline$V_{\min }$ & Minimal volume of lymphoid tissue (ml) & 100 \\
\hline$P_{0}^{*}$ & $\begin{array}{l}\text { Length of telomere repeats in naïve } \mathrm{T} \text { cells produced } \\
\text { at the age of } 18\left(\mathrm{bp} \mathrm{cell}^{-1}\right)\end{array}$ & $8.3 \cdot 10^{3}$ \\
\hline$P_{\text {min }}$ & $\begin{array}{l}\text { Minimal length of telomere repeats in the progenitor } \\
\text { of naïve } \mathrm{T} \text { cells }\end{array}$ & 100 \\
\hline$N_{0}$ & $\begin{array}{l}\text { The concentration of naïve } \mathrm{T} \text { cells at the age of } 18 \\
\left(\text { cell } \mathrm{ml}^{-1}\right)\end{array}$ & $1.9 \cdot 10^{9}$ \\
\hline$M_{0}$ & $\begin{array}{l}\text { The concentration of memory } \mathrm{T} \text { cells at the age of } 18 \\
\left(\text { cell } \mathrm{ml}^{-1}\right)\end{array}$ & $6.45 \cdot 10^{8}$ \\
\hline$P_{N 0}$ & $\begin{array}{l}\text { Length of telomere repeats in naïve } \mathrm{T} \text { cells at the age } \\
\text { of } 18\end{array}$ & $8.8 \cdot 10^{3}$ \\
\hline$P_{M 0}$ & $\begin{array}{l}\text { Length of telomere repeats in memory } \mathrm{T} \text { cells at the } \\
\text { age of } 18\end{array}$ & $7.4 \cdot 10^{3}$ \\
\hline
\end{tabular}

with the following initial conditions

$$
K\left(t_{0}\right)=K_{0} ; \quad C\left(t_{0}\right)=C_{m}+C^{*} ; \quad F\left(t_{0}\right)=\frac{\rho\left(C_{m}+C^{*}\right)}{\mu_{f}} ; \quad m_{c}\left(t_{0}\right)=0 .
$$

Note that the expression for initial value of specific lymphocytes contains the concentrations of naïve lymphocytes $C^{*}$ and memory cells $C_{m}$ that are specific to the pathogen under consideration. We assume that the level of specific naïve lymphocytes is homeostatic and in case of natural death of lymphocytes only the excess (cloned) lymphocytes die (see the last term in eq. 0.10).

The particular feature of the model of infectious disease is an equation for dynamics of target tissue damage. Wide tissue damage causes loss of homeostasis and reduces lymphocytes proliferation (the first term in eq. 0.10).

In clinical practice [23] a maximum of tissue damage is interpreted as disease severity $S$. In case of pneumonia damage of less than $15 \%$ cells of three lower segments of lung corresponds to mild disease with a low risk of death, damage of $35 \%$ cells of three lower segments of lung corresponds to medium 
TABLE 2. Initial conditions and model parameters for simulation of infectious disease

\begin{tabular}{|c|c|c|}
\hline Parameter & Physical meaning and dimension & Estimate \\
\hline$\beta$ & Rate of pathogen propagation $\left(\right.$ day $\left.^{-1}\right)$ & 0.35 \\
\hline$\gamma$ & $\begin{array}{l}\text { Rate of pathogen neutralization by antibodies (ml } \\
\mathrm{pt}^{-1} \mathrm{day}^{-1} \text { ) }\end{array}$ & $8.5 \cdot 10^{-14}$ \\
\hline$\alpha$ & $\begin{array}{l}\text { Rate of specific lymphocyte proliferation }\left(\mathrm{ml} \mathrm{pt}{ }^{-1}\right. \\
\left.\text { day }^{-1}\right)\end{array}$ & $5 \cdot 10^{-11}$ \\
\hline$\mu_{C}$ & Death rate of specific lymphocytes $\left(\right.$ day $\left.^{-1}\right)$ & 0.5 \\
\hline$\rho$ & Rate of antibody production by lymphocytes $\left(\right.$ day $\left.^{-1}\right)$ & $7 \cdot 10^{3}$ \\
\hline$\eta$ & $\begin{array}{l}\text { Number of antibodies required to neutralize one } \\
\text { pathogen particle }\end{array}$ & 20 \\
\hline$\mu_{f}$ & Death rate of specific antibodies $\left(\right.$ day $\left.^{-1}\right)$ & 0.05 \\
\hline$\sigma$ & $\begin{array}{l}\text { Rate of target organ damage by the pathogen }(\mathrm{ml} \\
\left.\mathrm{pt}^{-1} \text { day }^{-1}\right)\end{array}$ & $9 \cdot 10^{-9}$ \\
\hline$\mu_{m}$ & Rate of target organ regeneration $\left(\right.$ day $\left.^{-1}\right)$ & 0.4 \\
\hline$K_{0}$ & Infecting dose $\left(\mathrm{pt} \mathrm{ml}^{-1}\right)$ & $10^{3}$ \\
\hline$C^{*}$ & $\begin{array}{l}\text { The concentration of specific naïve lymphocytes (cell } \\
\mathrm{ml}^{-1} \text { ) }\end{array}$ & - \\
\hline$C_{m}$ & The concentration of specific memory cells $\left(\right.$ cell ml $\left.\mathrm{ml}^{-1}\right)$ & - \\
\hline$k_{1}$ & $\begin{array}{l}\text { The fraction of naïve } \mathrm{T} \text { cells involved in immune re- } \\
\text { sponse }\end{array}$ & $1.5 \cdot 10^{-6}$ \\
\hline$k_{2}$ & $\begin{array}{l}\text { The fraction of memory } \mathrm{T} \text { cells involved in immune } \\
\text { response }\end{array}$ & $4.3 \cdot 10^{-4}$ \\
\hline
\end{tabular}

severity with a death risk of 0.5 , disease with greater than $0.45 \%$ destroyed target tissue is defined as severe with a very high probability of death $(\geq 0.77)$.

Varying the rate of immune response and initial concentration of specific lymphocytes according to the age trend we can simulate the course of infectious disease for different ages. It is assumed that the concentration of specific lymphocytes at the beginning of the infection depends on current concentrations of naïve and memory $\mathrm{T}$ cells as follows

$$
C\left(t_{0}, \tau\right)=C^{*}(\tau)+C_{m}(\tau)=k_{1} N(\tau)+k_{2} M(\tau)
$$

where $t_{0}$ denotes the time of the beginning of disease, $\tau$ denotes the age.

There are a lot of evidence that the rate of lymphocyte proliferation decreases with age. Denote by $\alpha^{0}$ the rate of specific lymphocyte proliferation at age of 18 and by $\alpha(\tau)$ one at age $\tau$. We assume that the ratio $\frac{\alpha(\tau)}{\alpha^{0}}$ is equal to the ratio of replicative capacity of $\mathrm{T}$ cells involved in immune response at age $\tau$ to one at age of 18. The proliferative capacity of naïve T cells involved in immune response at age $\tau$ is defined by the concentration and the mean length of their telomeres $k_{1} N(\tau)\left(P_{N}(\tau)-H\right)$. Here $H$ is the Hayflick limit - the cell stops division when telomere becomes shorter than $H$. The proliferative capacity of memory $\mathrm{T}$ cells can be represented in the similar manner. Then, the expression for $\alpha(\tau)$ is as follows

$$
\alpha(\tau)=\alpha^{0} \frac{k_{1} N(\tau)\left(P_{N}(\tau)-H\right)+k_{2} M(\tau)\left(P_{M}(\tau)-H\right)}{k_{1} N^{0}\left(P_{N}^{0}-H\right)+k_{2} M^{0}\left(P_{M}^{0}-H\right)},
$$

where $P_{N}^{0}, P_{M}^{0}, N^{0}, M^{0}$ are values of respective variables for age of 18 .

Note that immune processes described in model of immunosenescence and in equation (0.10) are located in lymph nodes, equation (0.9) represents changes of pathogen concentration in target tissue and equation (0.11) deals with antibody concentration in the blood. We consider varibles located in different spatial compartments in order to have a right to use reasonable physical constants for modelling cell interaction. All transfer rates are comprised in the estimates for the rates of interactions (see for details [24]).

Acknowledgements. The authors thank Dr SG Rudnev for helpful suggestions and valuable discussions, This work is supported by the Max Planck Institute for Demographic Research in Rostock, Germany. 


\section{References}

[1] R. Aspinall. Longevity and the immune response. Biogerontology, 1(3) (2000), 273-78.

[2] A.G. Bodnar, M. Ouellette, M. Frolkis, et al. Extension of life-span by introduction of telomerase into normal human cells. Science, 279(5349) (1998), 349-52.

[3] G. Caselli, J.W. Vaupel, A.I. Yashin. Mortality in Italy: contours of a century of evolution. Genus, 41(1-2) (1985), 39-55.

[4] G. Caselli, R. Capocaccia. Age, period, cohort and early mortality: an analysis of adult mortality in Italy. Popul Stud (Camb), 43(1) (1989), 133-53.

[5] G. Caselli, E. Barbi. Italian mortality data by regions and causes of death. (1997) http://www.demogr.mpg.de.

[6] C. Caruso, S. Buffa, G. Candore, G. Colonna-Romano, D. Dunn-Walters, D. Kipling, and G. Pawelec. Mechanisms of immunosenescence. Immunity and Ageing, 6(1) (2009), 10.

[7] G. Caselli. The gender gap in survival: a new perspective. Ital J Gender-Specific Med, 2(2) (2016), 75-82.

[8] R.M. Cawthon, K.R. Smith, E. O'Brien, A. Sivatchenko, R.A. Kerber. Association between telomere length in blood and mortality in people aged 60 years or older. Lancet, 361(9355) (2003), 393-95.

[9] B. Choudhary, A.A. Karande, and S.C. Raghavan. Telomere and telomerase in stem cells: relevance in ageing and disease. Frontiers in bioscience (Scholar edition), 4 (2011), 16-30.

[10] R.B. Effros. T cell replicative senescence pleiotropic effects on human aging. Ann NY Acad Sci, 1019(1) (2004), $123-126$.

[11] R.B. Effros. Telomere/telomerase dynamics within the human immune system: effect of chronic infection and stress. Experimental gerontology, 46(2) (2011), 135-140.

[12] E.S. Epel, E.H. Blackburn, J. Lin, et al. . Accelerated telomere shortening in response to life stress. Proc Natl Acad Sci USA, 101(49) (2004), 17312-15.

[13] C. Franceschi, D. Monti, P. Sansoni, A. Cossarizza. The immunology of exceptional individuals: The lessons of centenarians. Immunol Today, 16(1) (1995), 12-16.

[14] C. Franceschi, S. Valensin, F. Fagnoni, C. Barbi, M. Bonafe. Biomarkers of immunosenescence within an evolutionary perspective: the challenge of heterogeneity and the role of antigenic load. Experimental gerontology, 34(8) (1999), 91121.

[15] A.A. Freitas, B. Rocha. Population biology of lymphocytes: The flight for survival. Annu Rev Immunol, 18(1) (2000), 83-111.

[16] T.R. Frieden. Can tuberculosis be controlled? Int J Epidemiol, 31(5) (2002), 894-99.

[17] T. Fulop, G. Dupuis, J.M. Witkowski, and A. Larbi. The role of immunosenescence in the development of age-related diseases. Rev Invest Clin, 2 (2016), 84-91.

[18] A. Herskind, M. McGue, N. Holm, T. Sorensen, B. Harvald, J. Vaupel. The heritability of human longevity: a populationbased study of 2872 Danish twin pairs born 1870-1900. Human genetics, 97(3) (1996), 319-23.

[19] M.R. Hilleman. Realities and enigmas of human viral influenza: pathogenesis, epidemiology and control. Vaccine. 20(25) (2002), 3068-87.

[20] Z. Ju, and K.L. Rudolph. Telomeres and telomerase in stem cells during aging and disease. In Genome and Disease Vol. 1 (2006), 84-103. Karger Publishers.

[21] B. Ljungquist, S. Berg, J. Lanke, G. McClearn, N. Pedersen. . The effect of genetic factors for longevity: a comparison of identical and fraternal twins in the Swedish Twin Registry. The Journals of Gerontology Series A: Biological Sciences and Medical Sciences, 53(6) (1998), M441-M446.

[22] G. Marchuk. Mathematical modelling of immune response in infectious diseases. Kluwer Academic Publishers, Dordrecht, 1997.

[23] G.I. Marchuk, E.P. Berbentzova. Acute pneumonia: immunology, severity assessment, clinic, treatment. Nauka, Moscow, 1989. (in Russian)

[24] G. Marchuk, R. Petrov, A.A. Romanyukha, G. Bocharov. Mathematical model of antiviral immune response. I. data analysis, generalized picture construction and parameters evaluation for hepatitis B. J Theor Biol, 151(1) (1991), 1-40.

[25] L. Mariani, G. Turchetti, C. Franceschi. Chronic antigenic stress, immunosenescence and human survivorship over the 3 last centuries: heuristic value of a mathematical model. Mech Ageing Dev, 124(4) (2003), 453-58.

[26] J.M. Murray, M.G. Law, Z. Gao, J.M. Kaldor. The impact of behavioural changes on the prevalence of human immunodeficiency virus and hepatitis C among injecting drug users. Int J Epidemiol, 32(5) (2003), 708-14.

[27] M.A. Murray and S.H. Chotirmall. The impact of immunosenescence on pulmonary disease. Mediators of inflammation, 2015(2015), http://dx.doi.org/10.1155/2015/692546

[28] G. Pawelec, E. Derhovanessian, A. Larbi, J. Strindhall and A. Wikby. Cytomegalovirus and human immunosenescence. Reviews in medical virology, 19(1) (2009), 47-56.

[29] G. Pawelec and E. Derhovanessian. Role of CMV in immune senescence. Virus research, 157(2) (2011), 175-179.

[30] S.R. Permar, W.J. Moss, J.J. Ryon, D.C. Douek, M. Monze, D.E. Griffin. Increased thymic output during acute measles virus infection. J Virol, 77(14) (2003), 7872-79.

[31] A.A. Romanyukha, A.I. Yashin. Age related changes in population of peripheral T cells: towards a model of immunosenescence. Mech Ageing Dev, 124(4) (2003), 433-43.

[32] T.E. Sannikova, S.G. Rudnev, A.A. Romanyukha and A.I. Yashin. Immune system aging may be affected by HIV infection: the mathematical model of immunosenescence. Russian Journal of Numerical Analysis and Mathematical Modelling, 19(4) (2004), 315-329.

[33] T.E. Sannikova. Analysis of infectious mortality by means of the individualized risk model. Mathematical modeling of biological systems. V. 2. Ed. Deutsch A. et al. Birkhäuser, Boston, 2008, 169-181.

[34] P. Sansoni, F. Fagnoni, R. Vescovini, et al. T lymphocyte proliferative capability to defined stimuli and costimulatory CD28 pathway is not impaired in healthy centenarians. Mech Ageing Dev, 96(1) (1997), 127-36.

[35] M.R. Sapin, L. Etingen. The Human Immune System. Meditsina, Moscow, 1996. (in Russian)

[36] P.E. Slagboom, S. Droog, D. Boomsma. Genetic determination of telomere size in humans: a twin study of three age groups. Am J Hum Genet, 55(5) (1994), 876-82. 
[37] G.G. Steinmann, B. Klaus, H.K. Muller-Hermelink. The involution of the ageing human thymic epithelium is independent of puberty. A morphometric study. Scand J Immunol, 22(5) (1985), 563-575.

[38] J.W. Vaupel \& A.I. Yashin. Heterogeneity's ruses: some surprising effects of selection on population dynamics. The American Statistician, 39(3) (1985), 176-185.

[39] J.W. Vaupel, Z. Wang, K.F. Andreev, A.I. Yashin. Population data at a glance. Odense Monographs on Population Aging, vol. 4. Odense University Press, Odense, 1997.

[40] J.W. Vaupel, J.R. Carey, K.Christensen, et al. Biodemographic trajectories of longevity. Science, 280(5365) (1998), $855-60$.

[41] K. Wu, N. Higashi, E.R. Hansen, M. Lund, K. Bang, K. Thestrup-Pedersen. Telomerase activity is increased and telomere length shortened in $T$ cells from blood of patients with atopic dermatitis and psoriasis. J Immunol, 165(8) (2000), $4742-47$.

[42] S. Zhao, Z. Xu, Y. Lu. A mathematical model of hepatitis B virus transmission and its application for vaccination strategy in China. Int J Epidemiol, 29(4) (2000), 744-52.

[43] J. Zhu, A.A. Quyyumi, J.E. Norman, et al. Effects of total pathogen burden on coronary artery disease risk and C-reactive protein levels. Am J Cardiol, 85(2) (2000), 140-46.

[44] J. Zhu, F.J. Nieto, B.D. Horne, J.L. Anderson, J.B. Muhlestein, S. E. Epstein. Prospective study of pathogen burden and risk of myocardial infarction or death. Circulation, 103(1) (2001), 45-51. 